\title{
\begin{tabular}{l|l} 
Mitraries & DSpace@MIT
\end{tabular}
}

\author{
MIT Open Access Articles
}

What is Language and How Could it Have Evolved?

The MIT Faculty has made this article openly available. Please share how this access benefits you. Your story matters.

Citation: Bolhuis, Johan J. et al. "What is Language and How Could it Have Evolved?" Trends in Cognitive Sciences 21, 8 (June 2017): P569-571 (c) 2017 Elsevier Ltd

As Published: http://dx.doi.org/10.1016/J.TICS.2017.05.007

Publisher: Elsevier BV

Persistent URL: https://hdl.handle.net/1721.1/128683

Version: Author's final manuscript: final author's manuscript post peer review, without publisher's formatting or copy editing

Terms of use: Creative Commons Attribution-NonCommercial-NoDerivs License 


\section{Letter \\ What is Language and How Could it Have Evolved?}

\author{
Martin B.H. Everaert, ${ }^{1}$ \\ Marinus A.C. Huybregts, ${ }^{1}$ \\ Robert C. Berwick, 2,3 \\ Noam Chomsky, ${ }^{4}$ \\ Ian Tattersall, ${ }^{5}$ Andrea Moro, ${ }^{6}$ \\ and Johan J. Bolhuis ${ }^{7,8, *}$
}

Unraveling the evolution of human language is no small enterprise. One could start digging somewhere in the largely unobservable past, working forwards to the present, hoping to surface in the right spot. Alternatively, one could start with the currently observed and well-established properties of human language, the phenotype of language, and work backwards, with these 'knowns' guiding the search for otherwise speculative historical 'unknowns'. In a recent issue of Trends in Cognitive Sciences, Corballis [1] appears confident that only the first strategy will serve. Evolutionary explanations necessarily are historical, but few evolutionary biologists faced with such a paucity of historical evidence would forge ahead without first defining what, exactly, the phenotype is that ultimately evolved [2]. Yet, Corballis criticizes what we actually know about the human language phenotype, because it does not conform to his speculations [3]. We believe that Corballis' odd research inversion suffers from misconceptions regarding what we know about both language and evolution.

\section{The Nature of the Human Language Phenotype (Is Not Communication)}

There is no denying that language is sometimes used to communicate, like this Letter. However, this should not lead to the apparently common fallacy that the design of language can be inferred reverse-engineering style from this single from watching Swan Lake. In the same functional perspective. Artful kinesthetics way, linguists now know with near surgiof human skeletal structures in motion, cal-precision how the sentence 'skeletal aka 'dance', also communicates, but structure' generally fixes meaning. We one would be hard pressed to derive know how 'communication' in the sense the evolutionary history of the human tibia of transfer of propositional meaning is

\section{Box 1. Structure Connecting Sound and Meaning}

Language is structured at all levels: phonology (sound structure), morphology (word structure), and syntax (phrase structure). The examples below show how structures built by the computational system are systematically mapped onto sound and meaning.

The two ways of pronouncing the string 'white board eraser', also reflected in spelling, are paired with a difference in their meaning: (i) whíteboard eraser: an eraser for whiteboards; and (ii) white bóard eraser: an eraser that is white.

The eraser in (i) itself could have any color, but in (ii) it has to be white. Both meanings and stress patterns are systematically derived from the structural patterns given in Figure IA,B, respectively.

In Figure IA, the adjective 'white' first merges with the noun 'board' and constructs the nominal compound 'whiteboard', which, as a unit, is merged with the noun 'eraser', yielding a bigger compound, an eraser, erasing what is written on whiteboards. When pronounced, the structure gives rise to the stress pattern [whíteboard eraser].

In Figure IB, 'board' first merges with 'eraser', producing the nominal compound 'board eraser', which, as a unit, is merged, in syntax, with the adjective 'white', yielding a nominal phrase, a board eraser that has a white color. When articulated (in speech), the structure gives rise to the stress pattern [white bóard eraser].

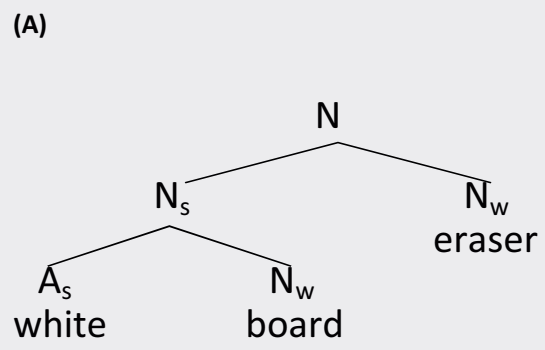

(B)

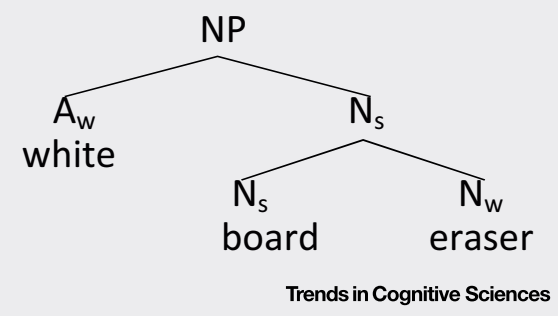

Figure I. Nominal compound (A) versus nominal phrase (B). Abbreviations: A, adjective; N, noun; s, strong; w, weak. 


\section{Trends in Cognitive Sciences}

facilitated by language: our manipulation of meaning is systematic and relies "on an ingrained ability to recognize structure in language." [4]. Meaning builds on a computational system that is sensitive to structural factors leading to hierarchical structure (Box 1). All this has been overlooked by Corballis.

'Language' reduced to a mode of communication becomes vacuous as an explanatory motivation. Bees communicate with flowers, your router communicates with your computer, we communicate via oil on canvas, and so on [5]. The Corballian world of communication is so diffuse that it becomes all the more puzzling why "the emergence of an organ as complex as language" would apparently be limited to humans.

\section{Structured Thought}

Corballis claims thought is structured, and that the "nature and structure of thought have a long and gradual evolutionary history", suggesting that, on this point, he is in agreement with the position advocated by Berwick and Chomsky [3]. However, all examples we know point to thought structured by syntax, not the reverse. The hierarchical structure built by the brain when processing sentences feeds our conceptual apparatus. Consequently, language is basically a thoughtexpressing tool $[3,6]$. For Corballis, 'thought' permeates the entire animal kingdom. That may be so, but he fails to give any clue as to what he might mean by this expansive notion of 'thought', how we could find out how ants, or songbirds 'think', and why, in this view, "expressive language" would take millions of years to appear.

\section{Recursion}

Corballis suggests that "generative grammar may [our emphasis] depend on the generative nature of spatiotemporal imagination, rather than on any property unique to language itself." However, this is merely idle verbiage. There is not even a remote connection between the two. If someone were to say that the waggle dance of the honeybee 'may' depend on the laws of motion, no one would pay attention. A recent comparative study of cross-species generative systems asserts that nonhuman animals have nothing resembling human recursive syntax [7]. While many animal species recognize statistical-probabilistic sequences, linear associations, or even algebraic rules, only humans appear capable of internalizing generative algorithms. In line with his view on 'spatiotemporal imagination', Corballis appears to assume that language inherits these sorts of property from similarly structured actions. Such an approach, linking recursion to observations from interactive language use, however, fails [8]. Moro has shown that the superficial parallels here between action sequences and sentences are misguided, again essentially backwards $[9,10]$. Selfreference, a defining property of recursion, appears to be absent from the domain of motor action and spatiotemporal imagination of nested maps [7], yet a rich part of human language.

\section{The Nature of Evolution}

We are surprised that Corballis subscribes to the view that anything other than an ancient and gradual origin for language "is counter to the theory of evolution", for he is clearly aware that our understanding of evolutionary mechanisms has been refined considerably over the past 150 years. Certainly, evolutionary change requires transitions from one viable state to another, but this does not entail that phenotypic steps are necessarily the tiny and incremental ones he favors. It is also unclear why he believes that changes in gene regulation cannot "add complexity", especially when it is almost certainly the case that modifications at this level gave rise to the anatomically distinctive species Homo sapiens [11]. Furthermore, the archeological record contains no evidence of behaviors compatible with the style of linguistic information processing until after anatomical Homo sapiens had come on the scene some 200000 years ago: a fact that Corballis mentions, but whose relevance is left undiscussed.

\section{Mental Time Travel}

Corballis also refers to, but regrettably does not discuss, the position that "No other organism, instantaneously and effortlessly extricates from the environment language-relevant data, and in a rather comparable way quickly attains rich linguistic competence, again a feat utterly beyond other organisms even in its rudimentary aspects." [12]. Corballis attributes these achievements to mental time travel (MTT) and Theory of Mind (ToM), although, as frequently noted, both of these competences are often dissociated from language ability: "autistic children highly defective in theory of mind [ . . . ] can acquire rich linguistic competence (and in fact a great deal of language acquisition proceeds before a child shows any sign of having attained theory of mind)." [12]. Significantly, again, no mechanism is suggested to lead from ToM to the specific structures of language. Worse, there is no discussion of what the mechanisms of recursive thought in MTT or ToM are, or how they lead to the feat that has to be explained. Shifting the burden from recursive language to recursive thought in $\mathrm{MTT}$ and ToM appears to us to leave the problem exactly where it was, adding nothing.

\footnotetext{
${ }^{1}$ Utrecht Institute of Linguistics, Utrecht University, 3512 JK Utrecht, The Netherlands

${ }^{2}$ Department of Electrical Engineering and Computer Science, Massachusetts Institute of Technology, Cambridge, MA 02139, USA

${ }^{3}$ Department of Brain and Cognitive Sciences,

Massachusetts Institute of Technology, Cambridge, MA 02139, USA

${ }^{4}$ Department of Linguistics and Philosophy,

Massachusetts Institute of Technology, Cambridge, MA 02139, USA

${ }^{5}$ American Museum of Natural History, New York, NY 10024-5192, USA

${ }^{6}$ School of Advanced Studies - Instituto Universitario di Studi Superiori, Pavia 27100 , Italy

${ }^{7}$ Cognitive Neurobiology and Helmholtz Institute, Department of Psychology, Utrecht University, $3584 \mathrm{CH}$ Utrecht, The Netherlands
} 


\section{Trends in Cognitive Sciences}

${ }^{8}$ Department of Zoology and St Catharine's College, University of Cambridge, Cambridge CB2 3EJ, UK

*Correspondence: j.j.bolhuis@uu.nl (J.J. Bolhuis). http://dx.doi.org/10.1016/j.tics.2017.05.007

References

1. Corballis, M.C. (2017) Language evolution: a changing perspective. Trends Cogn. Sci. 21, 229-236

2. Everaert, M.B.H. et al. (2015) Structures, not strings: linguistics as part of the cognitive sciences. Trends Cogn. Sci. 19, 729-743
3. Berwick, R.C. and Chomsky, N. (2016) Why Only Us: Language and Evolution, MIT Press

4. Winter, Y. (2016) Elements of Formal Semantics: An Introduction to the Mathematical Theory of Meaning in Natural Language, Edinburgh University Press

5. Moro, A. (2016) Impossible Languages, MIT Press

6. Chomsky, N. (2010) Some simple evo-devo theses: how true might they be for language? In The Evolution of Human Language (Larson, R.K., ed.), pp. 45-62, Cambridge University Press

7. Hauser, M.D. and Watumull, J. (2016) The Universal Generative Faculty: the source of our expressive power in language, mathematics, morality, and music. J. Neuroling.
Published online November, 9, 2016. http://dx.doi.org/ 10.1016/j.jneuroling.2016.10.005

8. Legate, J.A. et al. (2014) Recursive misrepresentations: reply to Levinson (2013). Language 90, 515-528

9. Moro, A. (2014) On the similarity between syntax and actions. Trends Cogn. Sci. 18, 109-110

10. Moro, A. (2014) Response to Pulvermueller: the syntax of actions and other metaphors. Trends Cogn. Sci. 18, 221

11. Tattersall, I. (2012) Masters of the Planet, Palgrave Macmillan

12. Chomsky, N. (2015) Some core contested concepts. J. Psycholing. Res. 44, 91-104 